\title{
Public Participation in the Development Process of a Mobility Assistance System for Visually Impaired Pedestrians
}

\author{
Nora Weinberger ${ }^{1, *(\mathbb{D})}$, Markus Winkelmann ${ }^{1}$, Karin Müller ${ }^{2}$, Sebastian Ritterbusch ${ }^{3,4}$ \\ and Rainer Stiefelhagen ${ }^{2,5}$ \\ 1 Institute for Technology Assessment and System Analysis, Karlsruhe Institute of Technology, \\ 76131 Karlsruhe, Germany; markus.winkelmann@kit.edu \\ 2 Study Centre for the Visually Impaired, Karlsruhe Institute of Technology, 76131 Karlsruhe, Germany; \\ karin.mueller2@kit.edu (K.M.); rainer.stiefelhagen@kit.edu (R.S.) \\ 3 iXpoint Informationssysteme GmbH, Pforzheimer Str. 160, 76275 Ettlingen, Germany; \\ sebastian.ritterbusch@ixpoint.de \\ 4 VWA-Hochschule für berufsbegleitendes Studium, Wolframstraße 32, 70191 Stuttgart, Germany \\ 5 Computer Vision for Human-Computer Interaction Lab, Karlsruhe Institute of Technology; \\ 76131 Karlsruhe, Germany \\ * Correspondence: nora.weinberger@kit.edu; Tel.: +49-721-608-23972
}

Received: 25 March 2019; Accepted: 16 April 2019; Published: 27 April 2019

check for updates

\begin{abstract}
Blind and visually impaired people have to cope with the safe movement through public space and the (lack of) knowledge of spatial issues and walkable routes. These challenges often lead to a fear of accidents and collisions, frequently also of disorientation. This, in turn, can result in a reduced radius of action, restricted mobility, and later on, in social isolation. Against this background, the project TERRAIN aims at developing a technical guidance system for orientation and navigation in urban space. For the development of this assistance system, the project pursues an approach in which reflexive, responsive, and deliberative dimensions have been integrated to address the ethical, legal and social implications (ELSI) in a co-design process. This paper focuses on the participation of citizens independent of vision impairments in the project which provided a variety of relevant indications of impacts and potential technical adaptations from an 'outer' point of view. In addition, conclusions can be drawn about the existing desirability and acceptance of the technical solution among the potential users as well as their social environment of potential users. In addition, it turned out that the citizen participation process raised different expectations among the project partners. Therefore, this article evaluates the participation results from the perspective of the technology developers and the technology assessors.
\end{abstract}

Keywords: grand challenge; people with visual impairments; technology assessment; demandorientation; public participation; co-design

\section{Introduction}

In 2015, the World Health Organization (WHO) estimated the worldwide number of people with some form of vision impairment at 1.3 billion. Of these, 188.5 million had mild and 217 million had moderate to severe vision impairments; 36 million people were blind $[1]^{1}$. Another 826 million people had a near vision impairment. By 2020, the share of persons affected will rise by a further 5.6 percent.

1 Some studies quote different figures because of other survey methods. 
The number of people with visual impairments in Germany is estimated at roughly half a million, with the number of those who are blind at approximately 100,000 [1]. This situation will probably be further exacerbated due to demographic change. The partial or complete loss of vision considerably restricts both the working and social everyday life of the affected persons. Therefore, they have to cope with two challenges: first, the safe movement through space and transport (navigation) [2], and second, the (lack of) knowledge of spatial issues and walkable routes (orientation) [3]. This is due to their problems with the perception of obstacles and landmarks that are crucial for generating a cognitive map to create an image of the surroundings and determine their own location. These challenges often lead to a fear of accidents and collisions, frequently also of disorientation [4,5]. This, in turn, can result in a reduced radius of action, restricted mobility, and later on in social isolation-three out of ten blind or visually impaired people never go outside without a (sighted) companion [6]. Another study showed that almost half of the participants had some problems in going out by themselves, with $20 \%$ of them citing fear or lack of confidence as reasons [4]. Among the elderly, the share is even higher-two in three do not leave their home anymore [7]. Likelihood of going outdoors decreases with age [4]. In our society, looking after impaired or elderly people is a strong moral obligation based on broad social consent.

This challenge is the starting point for the project on "Autonomous mobility for blind and visually impaired people in the urban space through audio-tactile navigation" (TERRAIN), which aims at generating structures targeted at the needs and interests of blind and visually impaired people for orientation in urban space. To this end, we develop a support and guidance system for orientation and navigation to strengthen the user's free movement, independence, and possibilities for interaction, and thus their social participation. The system does not replace traditional aids, such as the long cane, guide dogs, or orientation and mobility training, but rather complement them. At the same time, this reduces the risks in the context of orientation requirements significantly, especially regarding dangerous barriers (e.g., road traffic or obstacles in chest or head height). Using deep neural networks for image recognition and object localization, as well as information from digital Geo Information Systems, the proposed system analyzes the surroundings to inform the users about their current environment (see Figure 1 as an example). This can be done acoustically using ear-free bone-conducting headphones, haptically using a belt, wrist, or ankle bands, or tactilely using a mobile Braille device, to minimize the impairment of the remaining senses. The collection of information and the processing and output of navigation signals happens in real time to ensure the user's secure and independent movement in urban surroundings. Our unique feature is that the image processing developed in the project combined with environmental information, as well as haptic and acoustic ${ }^{2}$ transmission information, goes far beyond everything that is currently available on the market. Besides this, we deploy innovative software and hardware components, which consider risk parameters and landmarks to open up an individual urban spaces where affected people can move more safely. In this way, blind and visually impaired peoples' movement range can be extended by increasing their trust in the proposed system.

For the development of this assistance system, the project pursues an approach in which reflexive, responsive, and deliberative dimensions have been integrated in various modules.

2 Acoustic information means synthetic language, spatial sonification with artificial tones, spatial sonification with representative tones, use of Spearcons, Acusticons, and so on. 


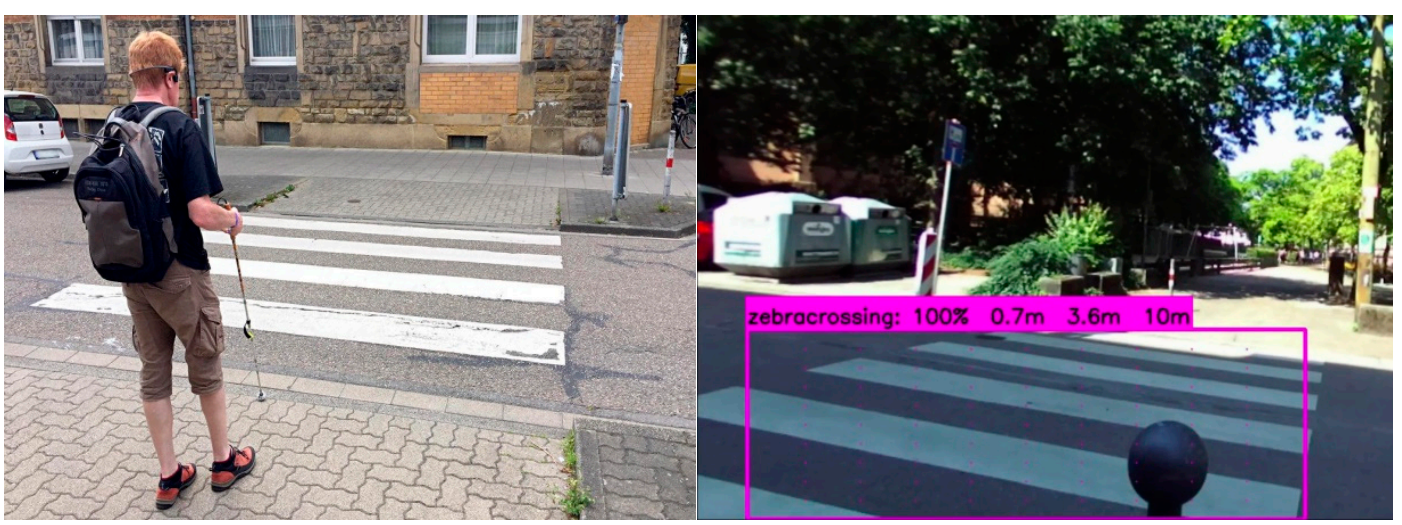

Figure 1. Detection of zebra crossings by analysis of the current environment ${ }^{3}$ (photographs: D. Koester, Screenshot).

Thus, people with blindness or vision impairments as well as mobility trainers are included in the technical development process in order to assure demand-oriented approach technology development in the sense of co-design - at the beginning of the project, a requirement analysis was carried out on current research literature on the needs of people living with blindness and vision impairment. In addition, mobility trainers were interviewed about mobility challenges in urban space for blind and visually impaired people. On the basis of this analysis, a first functional demonstrator was developed, which is tested and evaluated in a user study in the middle of the project. The results of this user test flow into the further development of the system. Finally, towards the end of the project there will be a second user ${ }^{4}$ study to evaluate the achieved status of the assistive technology ${ }^{5}$.

However, apart from the technical conditions, the project also has to consider and integrate ethical, legal, and social implications (ELSI) of innovation [8]. Ethical and societal questions are raised since the technology can cause implications for both the individual and the (urban) society. Therefore, the project also provides the framework for carrying out an anticipative reflection in the inter- and transdisciplinary dialogs of all concerned players and those potentially affected by the use of technology (like citizens ${ }^{6}$ ). The goal is to consider the respective expectations and perspectives and to be able to include potential controversies that might have otherwise been overlooked. This is achieved by technology assessment ${ }^{7}$ (TA, [9]) methodologies that accompany the complete technical development process; this is the establishment of a roundtable to moderate between the technical project partners and external project experts from ethical, economic, social, medical, and legal disciplines, as well as those directly affected by visual impairments or blindness also contributing their everyday life perspective. Several representatives of associations for the blind and visually impaired, an advisory board for people with disabilities, the municipal building authority and real estate office, mobility trainers, and other stakeholders are involved in deliberative processes. Mobility trainers contribute

3 The number indicates the probability assumed by the model that the object in the rectangle is a zebra crossing. Of course, the value 1.00 is only a rounding, there is never total security.

4 User, both here and in the further text, always means people living with blindness or vision impairments.

5 The term "assistive technology" in this article denotes any device or system that overcomes existing environmental and social barriers, thereby extending the opportunities and options open to disabled people, e.g., increases the ease and safety with which a task can be performed.

6 When we mention citizens in this article, we always refer to people who are not-impaired.

7 Technology assessment is a cross-disciplinary field of research that deals with scientific and technological change. The programmatic aim of TAs is to contribute to solving societal problems that relate to scientific and technological change. Accordingly, TA can be characterized as problem-oriented research that covers all consequences of technological applications in a comprehensive matter. Besides this, TA should provide knowledge about the anticipated consequences, in terms of time, early enough that the actors involved can recognize these consequences in good time and react to them with political measures. 
a special perspective here since they are directly involved in the technology development. Among other things (see above), they take part in the field tests in an advisory capacity.

In another part of the project, the values, needs, and expectations of citizens were confronted with the potential effects of research and innovation (R\&I) outcomes. The aim was to gather the acceptability of the technical innovation in a dialog and be able to improve it, if required. Potential changes of the social setting resulting from the utilization of technology, for example by wearing a camera in public spaces, were also discussed with the citizens in a workshop, based on narrative descriptions of the real life application situations. This allows forecasts on the question of which technology future should be advanced in the specific context according to the public mind. In this way, social imaginations of the technology future and its potential impacts are anticipated in the project.

All the results of these widespread participation processes are continuously integrated into the research process and are systematically and collectively reflected by the interdisciplinary project consortium. The strength of this approach is the continuous exchange between the technology developers and the outer world mediated by the TA work package. In addition, the results of these versatile participative processes are mirrored back to the involved stakeholders to realize continuous cross-referencing. This transparent and interactive process for capturing socially desired characteristics of technology and considering possible impacts offers the opportunity to develop a product that is considered acceptable by all stakeholders.

In the following, we present key findings of the citizen participation process, demonstrate how citizens can imagine a concrete technology future based on narratives, and list the social impact aspects they mention in the discussion of the matter. The results of the technology tests carried out with blind and visually impaired people and their assessment of the usability of the TERRAIN system are not part of this article; a separate publication is planned for this. In this article we derive an assessment of the evolving innovation from the citizen statements and then deduce an estimation of the societal acceptance of the evolving innovation. In the discussion, we reflect these results in light of the two perspectives of the technology developers and the TA, and argue from two positions in the project.

\section{Methodology}

\subsection{Acquisition of Workshop Participants}

In order to obtain a group of participants as diverse as current society, the local residents' registration office conducted a group sample for the selection of workshop participants. To this end, 3000 citizens of a Southern German city aged 18 or older were randomly chosen from group information provided by the responsible municipality ${ }^{8}$ and invited to the workshop. In response, 39 citizens officially signed up for the workshop ${ }^{9}$. The group consisted of 13 male and 26 female participants with a mixed age structure and a slight majority of attendees older than 50 . The youngest participant was 25 and the oldest was 87 years of age ${ }^{10}$. We have to point out here that we did not aim at a representative sample of the population, but wanted to capture the variety of potential chances and challenges that are seen by an interested citizenship, related to the technical innovation. As we did not want to attribute any allocation of the results to age, marital status, and educational level, these data were not collected. The age information mentioned above only serves to give the reader a "picture" of the group of participants.

8 According to paragraph 32 Meldegesetz Baden-Wuerttemberg (registration law), these data can be used for research with public interest. All data are handled according to data protection laws and were only used for this research project.

9 The random sample involved a citizen with sight impairment.

10 We do not provide any information on occupational or educational status, as this is irrelevant for the evaluation of the results in this article. 


\subsection{Workshop}

The starting point of all workshop discussions was to provide the citizens with a preferably neutral view of the project. Against this background, narratives provided the participants with the basic framework data required for discussion and (expected) technical opportunities, including the (intended) benefits for people with visual impairments. After this short introduction and the definition of the workshop goals, the first group task focused on the wishes and concerns related to technology use in public spaces. Participants worked on these questions using the so-called brainwriting pool method [10]. In three working groups, participants silently wrote down their thoughts on an empty index card. They passed their card on to their right table neighbor for additions, took another card, noted down other ideas, and handed this over to the next participant as well. Cards received from seat neighbors were read, complemented with own thoughts, and handed on like the card before. Once a card arrived back at the initial author, it was placed on the stack of cards in the middle of the table (pool). In this way, all the participants of the small group were able to gather and write down ideas on their own. More introverted participants had the same chance to provide and comment on their ideas as extroverted ones. Following this, the small groups discussed their ideas and tried to come to a consensus. In the last step, one participant of each group presented their results in detail to the rest of the plenum to allow for a workshop-specific weighting of the aspects discussed. This group task was followed by a module where participants could experience different forms of visual impairment by using simulating glasses which were provided by the German Association of the Blind and Visually Impaired. Some participants also decided to be guided through the room by a second person to get the feeling of "depending on someone" directly, which is often expressed by those affected. This exercise, which was very positively evaluated by the participants, was intended to raise awareness for the daily challenges of people with visual impairment. The citizens' experience was the starting point for another group task, where the previous work groups were split up and new groups were formed. The question for the second group task was: "Which possible positive and negative impacts of the technical innovation should be considered from your point of view?" This question was discussed in open, unmoderated groups, first from the citizens' perspective and in a second phase in the (anticipated) role of people with visual impairments. The results were then shared with the plenum again.

\subsection{Qualitative Content Analysis}

All the discussions during the workshop were captured by audio recording and note taking. After the workshop, we transcribed and anonymized all recordings. Participants were assigned an abbreviation code to respect their privacy. The analysis of the transcripts followed the qualitative content analysis method [11,12]. Categories were inductively chosen to analyze relevant aspects. As category construction needs particular attention $[10,12]$, the researchers intensively discussed and updated the categories in an iterative process to come up with meaningful and dense summaries of the discussed topics. For the coding of the material, "open coding" was used following a line-by-line procedure [13]. The outcome of this process is presented in the results section (see Section 4). Every category has several subcategories, each representing a topic that the listed statements referred to. An anchor citation for each category and subcategory provides a typical example for the character of the category [13]. Furthermore, a short summary was given for each category, also a list of (shortened) key citations, limiting the citations to key statements, as well as the list of full citations. As it was a strong intention to be in permanent dialogue with the citizens, the findings were mirrored back to the participants in form of a workshop report. Besides this, some individual interviews were carried out.

\section{Results of the Public Engagement Process}

In the framework of this article, we focus on the central aspects of the participation process. Order and length of topics in the individual groups play a minor role for the exploratory and qualitative topic selection. In the following descriptions, we differentiate the topics according to 
1. Technology-centered issues, which might need solutions mainly consisting of an adjustment and change of the assistive system's technical components; and

2. Non-technology-centered issues, which might call for additional measures apart from specific technical solutions.

We are well aware that this distinction is only due to practical reasons. Strictly speaking, technical and non-technical aspects cannot be separated. However, the distinction underline that the technical-centered issues have been directly incorporated in the process of technology development, while the social aspects have been taken into account in the design of the business model (consulting and information needs, etc.). In addition, some new research questions for follow-up projects and for the applications of obtained data in related humanitarian disciplines arose from the non-technology-centered issues. Some quotations ${ }^{11}$ are added for an even better illustration of the topics.

\subsection{Technology-Centred Issues}

Technology-oriented issues included visibility of technology, data collection and use, characteristics and expectations of technology, handling of technology, and additional features. These topics are described in detail below.

\subsubsection{Visibility of Technology}

The question of whether clear visibility of the assistive system's technical aids has more positive or negative effects was intensively discussed by the citizens. The discussion focused on the technology's signal function for other road users to enable them to better adjust to the situation and provide particular consideration or help, if necessary.

"Also the fact that you can see there is someone with a handicap, then I am more considerate than if I know there is someone just standing there ... of course, if I know he or she can see me, I drive by. If I notice that he or she is possibly not able to see me, I am more careful." (B1: 225-228)

"But, as I said, I was not able to recognize that she is blind, so, if I hadn't been strolling along, I wouldn't have noticed it. Someone is just standing there, and therefore it is important to recognize it, so that the other one, who can see, is indeed able to recognize it." (B3: 919-923)

Also an improved visibility at night was discussed. In general, the participants agreed on the assumption that the number of people with visual impairments who are on their way in the city will increase due to demographic change. One participant put it this way:

"The older our society becomes, the more visually impaired people we will have and the more natural it will probably become that these people do use these devices to make them more independent. And the more natural it might become for us to see people in the streets with their devices." (B3: 1013-1018)

Apart from that, citizens also fear that the use of technology could lead to a stigmatization of the users. In this context, they mentioned that both size and weight of the technical solutions should be as small, and thus as imperceptible, as possible.

\subsubsection{Data Collection and Use}

Various aspects of technical data collection to improve the orientation of people with visual impairments have been topics of the citizen discussions. First, it was discussed that "(we have to consider) which data we need to realize the system. And then we have to weigh up, if we collect data, where and how do we

11 The coding describes in which of the three groups of citizens the statements were made (group of citizens $1=\mathrm{B} 1$, etc.). The line numbers of the respective transcripts are also indicated. In addition, it should be noted that the statements were not personally identifiable, as this was not essential for this article. 
store them?" (B1: 627-641). Especially, the use of cameras in public spaces and the related evaluation options were greeted with skepticism. The general requirements for sensible use of image recognition and processing include the exclusive use for orientation purposes, the timely deletion of collected data, the exclusion of automated recognition of vehicles or persons, and the exclusion of the disclosure of the collected data to third parties (e.g., regulatory authority, police, and companies). According to the participants' views, data protection (i.e., access to data) and data security (the exclusion of data manipulability) should be guaranteed by local data processing, i.e., without internet connection (technical isolated application).

\subsubsection{Characteristics and Expectations to Technology}

Apart from the characteristics already mentioned regarding visibility and weight of the assistive system - "then we also had that the devices should become so small that they ... do not catch someone's eye" (B1: 321-322)—citizens expressed further wishes as technology requirements during the discussion. They requested that the technical system's acoustic signals have to be clearly distinguishable from possible surrounding noises. The discussants also considered the volume of signals, for both the user and the direct social environment. These signals should be as discreet as possible, so that they do not interfere with, or drown out, warning shouts or other social interactions. Besides this, the participants referred to the increased importance of hearing for spatial orientation in combination with visual impairments, which should not be affected by technical signals. In general, the technical system should report obstacles or dangers immediately and directly to allow the users reaction in time to change their direction, for example. Nevertheless, the type or volume of signals should not startle the users.

The participants also requested that the technical system should be highly compatible with other technical devices and applications to make sure that the users are not bound to one or a few providers of technical components. Also, the possibility of connecting different devices to increase the number and quality of information about the surroundings was pointed out. Traffic signal controls, for example, could provide useful information for a "certain spatial orientation" (B1: 747-750). Communication with (semi)autonomous cars was also intensely discussed, "That the [affected people] can make contact with approaching cars, etc., and the other way round" (B2: 1069-1076). Citizens also exchanged their views on a user network for the proposed system. This could make the system self-learning, for example by using the routes of other affected people. The existence of dead spots and insufficient signal reception, especially in rural areas, was also addressed:

"GPS is also not so precise yet, I think $10 \mathrm{~m}$, and in many places you don't have it, especially if I'm out in the countryside ... so, when I'm doing my trekking tours, I have walked really strange ways. Well, the infrastructure is not that good yet, so even if I do have the device, this does not automatically include the infrastructure." (B2: 661-667)

For the cases of technical system failure, redundancies were considered appropriate, i.e., the possibility to switch to other systems or system components with equivalent functions. In this context, existing solutions, such as the long cane, were mentioned as well. An easy and fast change of support systems improves security and increases the users' options.

\subsubsection{Handling of Technology}

Regarding the simple and intuitive handling of technical components, especially for elderly users, the participating citizens called for an option to customize the technical system, i.e., adapt its feedback to the individual users and their special requirements. Some participants even suggested specific technical solutions:

"Then it only has to be designed in a way that allows elderly people with slightly shaking fingers to use it, too. With real buttons and not these touch keys the iPhone uses, but something to press, that could make sense." (B3: 1068-107) 
In the participants' view, the need to make themselves familiar with a smartphone could keep elderly people from using it. Nevertheless, opposing opinions were also expressed, since the number of smartphone users is also growing among the older generation. However, they agreed that the integration of smartphones as the central interface between people and individual technical components could definitely be a feasible method. Apart from that, as a certain contradiction to the system's individual configurability, it was requested that the number of necessary technical components should be as small as possible, i.e., few devices with many functions rather than a large number of specialized single devices.

The participants themselves came up with the possibility of implanting (neuronal) chips to improve the human-machine interface, which provoked wide and controversial discussions. While some participants considered such a physical intervention as possible and beneficial, there were also determined opponents of such a scenario.

\subsubsection{Additional Features}

An interesting new perspective arose from the participants' idea to not only provide the users of the proposed system with the immediate orientation function, but also with additional features. Aside from additional information about the direct surroundings (such as logical abbreviations and timetables, which could be provided online), the question "how it will look like in the dark, just thinking of the camera" (B1: 290-291), was discussed. Such features would not only compensate for the lack of eyesight, but would, according to the participants, also provide additional options, which could be interesting for sighted people, too. This could result in a wider dissemination of the system under development, beyond its niche function as an orientation aid for people with visual impairments.

\subsection{Non-Technology-Centered Issues}

Among more technological debates, a number of discussions were centered on societal impacts-potential for social participation, financing and costs, fears and dangers, characteristics and skills of users, liability, as well as freedom and independence.

\subsubsection{Potential for Social Participation}

According to the citizens' view, mobility is a fundamental prerequisite for participating in social activities. However, from their perspective, the technical options to improve the mobility of visually impaired people can also influence the social interaction within the cities and in our general coexistence. Being in the role of those affected, they argued as follows:

"I'm only handicapped if I can no longer participate in social life. But as soon as I can participate in social life with the help ... of my devices, I would say I would not really feel like being handicapped." (B3: 1109-1112)

"On the other hand ... technology could also make people start a conversation or provide them with other ways of communication than before—or make them a bit more integrated." (B2: 540-544)

Encouraging the integration of those affected into the job market was also mentioned as a promising impact of technology innovation:

"Even though I'm blind, I want to continue to be a working woman. I do not only want to sit at home or go for walks." (B3: 1086-1087)

\subsubsection{Financing and Costs}

A recurring topic discussed in the different groups was the financing of the assistive system. While technical orientation aids for visually impaired people were favored by the majority, opinions regarding the occurring costs differed. The participants agreed that the financial responsibility for 
the basic technical equipment for those affected should lie with the health insurance companies, for example by including the orientation aid in their technical aids register. However, the technical components and especially the range of functions of such basic equipment were intensively discussed. The extent to which improved orientation aids for the visually impaired could also lead to an additional financial burden, for example in the form of higher health insurance contributions or taxes, could not be discussed in detail due to the tight workshop schedule. However, in the participants' view, this remains a relevant question, since it might be a potential impact of technology development on society.

"We will ... of course, there are people who can pay for this, but there is also a large group who cannot afford it and then the community has to pay for it as well, and where does this end?" (B2: 577-581)

In this context, a certain consensus might be carefully phrased like this: according to the citizens, the costs for improved orientation possibilities for visually impaired people should be seen as a necessary and legitimate contribution which society has to make, while potential additional benefits have to be financed by the respective users.

\subsubsection{Fears and Dangers}

There was also a varied and vivid exchange about the fears and dangers possibly arising from the use of the new technology. The technical enhancement of the possibilities of visually impaired people could also have negative consequences for the users or their social environment-or even lead to exclusion. One issue addressed in this context was changes in direct social interaction patterns that might occur when people do not know what possibilities a user of the proposed system has, and in which situations help is still needed or desired. Thus, there is a potential danger of reduced helpfulness of passers-by resulting from an overestimation of the technical aids. Another potential negative effect, according to the participating citizens, could be reduced social contacts and interactions. People might be prevented from posing a short question, giving a well-intentioned hint, or other kind of communication if they fear that the approached person would be disturbed or even distracted from the technology, and thus miss important signals. This insecurity in dealing with those using the orientation aid should be counteracted by providing information. Two representative citizen statements in this context are:

"Human contact in helping situations gets lost." (B3: 143)

"That he does things and realizes that the more he helps himself, so to speak, the less he relates to others. Because it's just possible for him to do it. And if . . a aids are developed, this does not help-not even if politics create a sort of great openness in society." (B2: 647-652)

Distraction from the concrete situation through the technology was seen as another potential danger. "Too much technology, too many sensory impressions, it gets too much, this can confuse me" (B2: 20-21). It could also be critical if the proposed system demands the user's attention in any situation, although there is already enough information available, or even worse, if the technology drowns out important environmental information (e.g., warning shouts or sirens). Possible solutions discussed were an emergency switch to immediately turn off the sound or an emergency call button, which automatically sends an alarm call, for example, in the event of a failure of technology.

In addition, a general concern of the citizens was that users of the system could sooner or later become dependent on the technology by losing — or at least not regularly practicing-their ability to orient themselves independently from the technological system:

"Now this is my point, which I've just formulated, that, for heaven's sake, I would not want to be dependent on technology. It should help me, help as inconspicuously as possible, but I do not want to become dependent on it." (B1: 608-612)

Another danger from the social environment was seen in possible desires the technology might create. Depending on the features and visibility of the camera system, the use of the proposed system 
could, according to the participants, provoke envy or even theft. Purely technical countermeasures, such as voice recognition or fingerprint sensors, would only be of limited use, because to be effective the security instruments would have to be recognized by others, which in turn, might even increase their desire and interest.

\subsubsection{Characteristics and Skills of Users}

Besides the features and options of the proposed technology, the skills needed by the users were also discussed in various contexts. The user must not only be confident in handling smartphones but also willing and able to configure the system. The customization and personalization of the components, in particular, requires practice with the software, according to the participants. Moreover, there is a need for guided training in the use of the individual system components and their interaction in order to take full advantage of the benefits of the proposed system.

\subsubsection{Liability}

The question of liability addressed in the group discussions was mainly about the regulation of possible responsibilities regarding the use of the system. With reference to the current debate in Germany about autonomous vehicles, the participants raised the question of the accountability of different stakeholder groups, such as pedestrians, insurers, car manufactures, motorists, and cyclists. The option of extended use of data automatically collected by the system in public spheres was declined throughout the discussions with reference to data protection.

\subsubsection{Freedom and Independence}

The discussion also included the possible effect of greater freedom for the system users. The technical system was expected to increase independence from human assistance and the respective schedules. In the view of the participants, the system would enhance the ability of visually impaired people to independently decide on the time and place of their activities in public spaces. This aspect of self-determination was unanimously considered positive in the discussions and seems to be an ideal goal worth striving for from the citizens' point of view.

\section{Discussion}

As the project showed that citizen participation partly raised different expectations among the project partners, therefore, we will argue in the following discussion from two positions in the project: on the one hand, from the perspective of the technology developers, and on the other hand, from that of the TA.

\subsection{The Technology Developers' Perspective}

It has become evident that the integration of citizens is also highly relevant in the technical development of an assistance system for blind and visually impaired people in urban environments. Since the system has a direct impact on the interaction and coexistence of blind or visually impaired and sighted people, it is essential to consider the conditions and effects in the design and implementation process. In contrast to the usage tests, where the technology is tested by future users, the public participation process offers the opportunity to look at the technology from an outside perspective. For technology developers the external view of citizens offers insights into the effects and the acceptance of the technical solution by citizens, allowing them to adapt the technology, take into account new aspects, consider possible future reactions by the urban population, and develop appropriate solutions in the innovation process.

The outside perspective of the participating citizens in the TERRAIN project offered many insights into non-technical and technical aspects directly or indirectly related to the design and impact of the technology. Of particular interest were issues linked to fears and dangers: 
- $\quad$ acceptance (stigmatization, limits)

- dependence on technology

- interaction with the technology used (training)

- social interaction

- envy

- payers

This shows that the discussions looked at the technology from very different angles, some of which had initially not been the focus of technology development. The issues addressed included operation and use concepts, financing of the solution, aspects of care and social interaction in the use of the technology, technical aspects, such as night vision, as well as emotional factors, such as envy, worries, and fears. A surprising result for the technology developers was that the use of a camera system was viewed far less critically than previously suspected. Therefore, they can increasingly work towards an integration of the visual components.

The participating citizens also mentioned aspects that had already been considered in the project planning. For example, in the debate, the citizens pointed to the need for technical training, which was already addressed by the planned training modules of the project. Such examples can be seen as confirmation of both the project itself and the technological solution: they clearly indicate that development is guided in an ethically and socially acceptable direction, which is highly important for successful market entry. For example, if the participating citizens had revealed a strong rejection of the use of cameras, technology development would have to account for this aspect, perhaps by emphasizing the system's privacy protection or the fact that there is no surveillance function included. It would also be necessary to clarify that the project had been approved by data protection authorities to ensure the data are processed in compliance with data protection laws. Depending on the feedback, it might have been possible to develop alternative variants with and without camera use. Moreover, the results provided valuable information for the public presentation of the services. Although, of course, the focus is on the group of the blind and visually impaired, these persons are accompanied by many sighted people who need to be equally informed about the products and services offered.

The described deliberative process also revealed some aspects that will only become relevant in subsequent projects or at later stages of development (e.g., the technology's approval as an aid, and associated therewith, cost coverage by health insurance carriers).

It is important for technology developers to be aware of the differences between technology development, usage studies, and citizen participation. This is because the expectations of the results of public participation were, and are, of a significantly different nature. In some cases, it is still necessary to develop further methods to transfer non-technical results of such participatory processes directly in the technology development. These results are important even after the project for the development of business models. Furthermore, there will always be some aspects that are not covered in public participation but are still relevant to the project, such as the inexpensive use of the proposed technology as a smartphone app compared to other tools, reduced risk of stigmatization, and possible use of the app not only by blind people but also by a broader user group.

Another point to be questioned is the timing of citizen participation in the course of the project. Since the results of the participation process have only been available at a later stage of the project, they can hardly be considered in the already very advanced technology development. Citizen participation at the beginning of the project, followed by an initial evaluation, would have better accounted for the results in the planning and technology development. Nonetheless, in view of the expected results, participation at the later stage of the project can still have an important impact on further development for market entry following the project. 


\subsection{The TA Perspective}

From the TA perspective, the results provide evidence that the deliberative approach-here consisting of the participation of an interested urban society and the analysis of the predominantly consensual attitudes of citizens toward the assistive system for blind and visually impaired people-can provide a deeper understanding of the acceptability of future technologies, such as the proposed system. The results also confirm that the citizens' contributions to the discussion help account for the possible implications of the dissemination and use of the new product in the prospective analysis of the innovation path [14]. Major socio-technical consequences identified by the citizens are related to:

- provision of opportunities for social participation;

- exclusion of those affected;

- fears and dangers associated with the proposed system;

- financing and costs;

- user characteristics and capabilities;

- liability; and

- freedom and independence of visually impaired people.

The list shows that the identified perceived chances and risks of the potential futures of a concrete technology are largely systemic and that interactions between the features of this complex technology are considered in their social context [15]. One example is the intensive debate among citizens on the risk of exclusion of people by using the technology in public spaces.

Against the background of the findings, our research question of whether it is possible for the interested public to imagine desirable technical solutions geared to the needs of the technology users can be answered positively. Furthermore, we demonstrated how to initiate and achieve a discussion about an acceptable balance of interests between potential technology users and their social environment. The narratives developed for the use of technology in public urban spaces have proven to be constructive. On the one hand, they made it possible to convey the use contexts, and on the other hand, enabled the discussion about the potential of a concrete scientific-technical solution. The workshop participants were enabled to imagine relevant situations of blind and visually impaired people, i.e., the action context. They were also able to assess whether the technology has to be regarded as helpful or desirable-sometimes by taking the perspective of those affected. ${ }^{12}$

The participants were not able, nor intended, to discuss specific technical matters. This was neither necessary, nor strived for, in the participation process, since the usability evaluation and fine-tuning of technical details is the subject of the further user tests. From a general perspective, these results enable specific social "fine-tuning" of the technical solution and its societal introduction. Citizens were able to explicitly envision technical features and functionalities on a more general level, as the variety of the above-mentioned technical aspects proves. Also, the potential social impacts associated with the use of the technology were widely discussed. The findings, thus, provide evidence of the fertility of deliberative formats to discuss technical potentials in connection with specific social needs of social subgroups (e.g., blind and visually impaired people), compared with desires, requirements, and needs of people who are (initially) not affected. These non-technical issues will be taken into account in the design of business models (consulting and information needs, etc.). In addition, some new research questions for follow-up projects and for the applications of the obtained results in related humanity disciplines arose from these non-technically-centered issues.

In addition, it can be shown that besides the described technical and socio-technical consequences, the discussions were suitable to identify acceptance criteria for new technological developments. One example was that the discussion on the financing of the assistive system implicitly revealed some

12 Of course, the valuations made are still those of people who are not affected. 
acceptance criteria and thresholds, such as the costs "would certainly lead to increased health insurance contributions for all" (B2: 577).

However, for the projection of social consequences, the methodological challenge is to translate the empirically collected arguments in such a way that technology developers can use them as the basis of their considerations. To make the non-technical requirements for technology developers more tangible, a kind of translation tool-so-called Action Sheets-was used, which was further developed on the basis of a previous study [16]. Nevertheless, it still seems to be difficult for technology developers to fruitfully use the non-technical results of such participation processes in defining their own role and responsibility.

Furthermore, it can be stated that a format aligned to a specific problem-which can be attributed to one of the grand challenges, namely health, demographic change, and wellbeing-can be realized if the corresponding social action context is successfully addressed. A "real" experience of the problems seems to further enhance this success, e.g., the experience of blindness through simulation goggles as used in the citizen workshop. The results of the participation process seem to confirm this, since the participants agreed on a positive assessment of the technical solution's potential. Therefore, we can assume that the narrative descriptions of the situation made the functional benefits of the technology more visible and perceptible, and thus contributed to increased ethical and social acceptance.

The results also show the challenges in adapting research results from a deliberative process, which is partly challenged by limited funding period and lack of flexibility in the course of the project. In order to exemplify this, we draw on the aspect of exclusion or inclusion of technology, which was strongly emphasized by the citizens. As mentioned above, they did see the high potential of the proposed innovation, but they also stated a risk of exclusion, e.g., through the visible ${ }^{13}$ use of the technology. This aspect needs further research. Due to the time and content requirements and limited funding period, this is not feasible within the presented project.

\section{Conclusions}

Our study provides insights on the current application of anticipative, reflexive, responsive, and deliberative methods as components of the specific project context.

The citizens' views on the technical advances brought a broad variety of relevant references to impacts and possible technology adaptations. Moreover, we can draw conclusions to the existing desirability and acceptability of the technical solution. Actually, we could not find any rejection of the technical innovation among the participating citizens. On the contrary: the use of the camera system was viewed far less critically than previously expected. The potential of the proposed system for the inclusion of blind and visually impaired people through increased mobility in the urban space is assessed as very high. The general idea of inclusion as a goal of technology development was highly appreciated by all participants.

The comparison of the perspective of TA researchers and technology developers demonstrates the challenge of dealing with transdisciplinary input in an interdisciplinary consortium. One needs to be aware of different expectations of the results from citizen participation processes. This can be illustrated by a metaphor from fishing-Harpoon fishing versus net fishing. Technology developers phrase their expectations with a focus on acceptance and technology in an efficiency-oriented way; they want to catch the big "technology fish" with a harpoon. A deliberative or explorative TA, in contrast, wants to get the "by-catch from the sea of technology impacts" with a net, since this "by-catch" might hide potential ELSI treasures. You can hardly catch a non-technical fear of exclusion with an engineer's "technical specification" harpoon; this is much more likely with the by-catch in the TA net. It is exactly this by-catch that allows the identification of social acceptance issues of technology use by including thematic "fish" that have been unnoticed so far.

13 The goal is to make the technology as invisible as possible, but this requires further advances in miniaturization. 
The discussion also indicates a potential need for accompanying research tasks in technical development projects. Reflexive and responsive up- and downstream loops and processes are necessary and lead to social acceptance. However, due to the high level of maturity of the technical solution in question, this was not possible in a three-year project with rather defined structures. The number of degrees of freedom for adaptations in the course of the project and in technology development was considerably reduced. From the perspective of technical development, efficiency could increase with more space for alternative socio-technical considerations. This would require pre-projects, which do not start with a specific technical idea, but with a collection of needs in the targeted social context. Projects should start with a phase of deliberative processes, followed by a specific technology development project, such as those funded in the call for so-called "Preliminary projects" of the German Federal Ministry of Education and Research. The next step would be the scientific accompaniment of every-day technology use to assess the actual lifeworld effects, in our example the impacts of exclusion, and especially, inclusion. In this step the same group of citizens should also be involved again. Here it would be interesting to analyze the evaluations from a theoretical external viewpoint with the evaluations from the viewpoint of a real use of technology.

Overall, we can state that the successful citizen participation process in the described project provides an essential contribution to the social, legal, and ethical compatibility of technical solutions. Moreover, it proved that the proposed system is basically accepted by the participant group, since the citizens as representatives of an urban society did not find any general negative impacts.

Author Contributions: Conceptualization: N.W., M.W., K.M., S.R., and R.S.; methodology: N.W., M.W., K.M., S.R., and R.S.; investigation: N.W., M.W., K.M., S.R., and R.S.; resources: N.W., M.W.; writing-original draft preparation: N.W.; writing-review and editing: N.W., M.W., K.M., S.R., and R.S.; project administration: S.R.; funding acquisition: K.M., S.R., R.S., and N.W.

Funding: The German Federal Ministry of Education And Research, grant number 16SV7611, funded this research.

Acknowledgments: The authors wish to thank the entire consortia of the project, "Independent mobility of blind and visually impaired people in urban space through audio-tactile navigation", in which the results presented here were gathered. Above all, we would like to thank the citizens for their commitment during the workshop. Without them, transdisciplinary research would not have been possible; therefore, everyone has made a decisive contribution to the success of the project to date. Thanks also go to the German Federal Ministry of Education and Research (BMBF) for promoting our research and innovation.

Conflicts of Interest: The authors declare no conflict of interest. The funders had no role in the design of the study; in the collection, analyses, or interpretation of data; in the writing of the manuscript, or in the decision to publish the results.

\section{References}

1. Bourne, R.R.A.; Flaxman, S.R.; Braithwaite, T.; Cicinelli, M.V.; Das, A.; Jonas, J.B.; Keeffe, J.; Kempen, J.H.; Leasher, J.; Limburg, H.; et al. Magnitude, temporal trends, and projections of the global prevalence of blindness and distance and near vision impairment: A systematic review and meta-analysis. Lancet Glob. Health 2017, 5, e888-e897. [CrossRef]

2. Petrie, H.; Johnson, V.V.; Strothotte, T.; Fritz, S.; Michel, R.; Raab, A. MOBIC: Designing a Travel Aid for Blind and Elderly People. J. Navig. 1996, 49, 45-52. [CrossRef]

3. Brabyn, J.A. New Developments in Mobility and Orientation Aids for the Blind. BME 1982, 29, $285-289$. [CrossRef] [PubMed]

4. Pavey, S.; Dodgson, A.; Graeme, D.B. Clements: Travel, Transport, and Mobility of People Who are Blind and Partially Sighted in the UK; Final Report for the RNIB; University of Birmingham: Birmingham, UK, 2009.

5. Manduchi, R.; Kurniawan, S. Mobility-Related Accidents Experienced by People with Visual Impairment. J. Res. Pract. Vis. Impair. Blind. 2011, 4, 44-54.

6. Clark-Carter, D.D.; Howarth, C.I.; Heyes, A.D.; Dodds, A.D.; Armstrong, J.D. The Visually Handicapped in the City of Nottingham: A Survey of Their Disabilities, Mobility, Employment and Daily Living Skills; University of Nottingham: Nottingham, UK, 1981.

7. Yerasimuo, N. Travellers' Tales-Making Journeys Safer; Royal National Institute for the Blind: London, UK, 2002. 
8. Zwart, H.; Landeweerd, L.; van Rooij, A. Adapt or perish? Assessing the recent shift in the European research funding arena from "ELSA" to "RRI". Life Sci. Soc. Policy 2014, 10, 674. [CrossRef] [PubMed]

9. Grunwald, A. Technikfolgenabschätzung-Eine Einführung; Gesellschaft-Technik-Umwelt: Berlin, Germany, 2010.

10. Schlicksupp, H. Ideenfindung: Innovation, Kreativität und Ideenfindung; Vogel Buchverlag: Darmstadt, Germany, 1992.

11. Mayring, P. Qualitative Content Analysis. Forum. Qual. Soc. Res. 2000, 1, 2.

12. Mayring, P.; Fenzl, T. Qualitative Inhaltsanalyse. In Handbuch Methoden der empirischen Sozialforschung; Baur, N., Blasius, J., Eds.; Springer Fachmedien: Wiesbaden, Germany, 2014; pp. 543-556.

13. Mayring, P. Qualitative Content Analysis: Theoretical Foundation, Basic Procedures and Software Solution; gesis Leibniz-Institut für Sozialwissenschaften: Klagenfurt, Austria, 2014; 143p.

14. Grunwald, A. Assigning meaning to NEST by technology futures: Extended responsibility of technology assessment in RRI. J. Responsib. Innov. 2017, 4, 100-117. [CrossRef]

15. Hellström, T. Systemic innovation and risk: Technology assessment and the challenge of responsible innovation. Technol. Soc. 2003, 25, 369-384. [CrossRef]

16. Kunz, A.; Pohlmann, S.; Heinze, O.; Brandner, A.; Reiß, C.; Kamradt, M.; Szecsenyi, J.; Ose, D. Strengthening Interprofessional Requirements Engineering Through Action Sheets: A Pilot Study. JMIR Hum. Factors 2016. [CrossRef] [PubMed]

(C) 2019 by the authors. Licensee MDPI, Basel, Switzerland. This article is an open access article distributed under the terms and conditions of the Creative Commons Attribution (CC BY) license (http://creativecommons.org/licenses/by/4.0/). 